\title{
Screening of Several Anti-Infectives for in Vitro Activity against Mycobacterium smegmatis
}

\author{
Grace Lovia Allotey-Babington1,2, Henry Nettey', Philip Debrah1, Ofosua Adi-Dako1, \\ Clement Sasu1, Anastasia Antwi', Yvonne Darko', Newriza Nartey'1, Jida Asare ${ }^{1}$ \\ ${ }^{1}$ University of Ghana School of Pharmacy, Legon, Ghana \\ ${ }^{2}$ Mercer University School of Pharmacy, Atlanta, GA, USA \\ Email: hnettey@msn.com, hnettey@ug.edu.gh
}

Received 19 October 2014; revised 20 November 2014; accepted 9 December 2014

Copyright (C) 2014 by authors and Scientific Research Publishing Inc.

This work is licensed under the Creative Commons Attribution International License (CC BY). http://creativecommons.org/licenses/by/4.0/

(c) (i) Open Access

\begin{abstract}
Aim: To evaluate in vitro the effectiveness of several anti-infective agents alone or in combination against Mycobacterium smegmatis. Method: A convenient stratified sampling method was used to obtain selected anti-infective agents. For individual drug samples, Minimum Inhibitory Concentrations (MIC) were obtained using the agar-well plate diffusion technique. Fractional Inhibitory Concentration Indices (FICI) were calculated for drug combinations using their MIC as obtained from the broth dilution method. Results: Of the thirty (30) anti-infective agents analyzed, ten (10) had MIC equivalent to or better than rifampicin (reference TB drug). Seven (7) drugs had MIC higher than rifampicin, while twelve (12) showed no growth inhibition of $M$. smegmatis. Analysis of the effect of drug combinations on $M$. smegmatis indicated that four (4) combinations, including rifampicin/ethambutol showed synergism. One (1) was additive, two (2) were indifferent and one (1) combination showed antagonism. Conclusion: Notable in the results obtained was the high effectiveness of the carbapenems in inhibiting the growth of $M$. smegmatis. Carbapenems, though not indicated for TB treatment, has a potential of playing a significant role in the treatment of tuberculosis. Also the drug combinations which showed synergism, especially those that involved the macrolide antibiotics, should further be investigated. These results have to be confirmed by in vivo clinical studies to define their roles in tuberculosis treatment.
\end{abstract}

\section{Keywords}

Antimicrobials, Minimum Inhibitory Concentration, M. smegmatis, Tuberculosis 


\section{Introduction}

Tuberculosis (TB) is an infection ultimately caused by the Mycobacterium tuberculosis (MTB) that is spread from person to person through airborne particles [1]. TB of the lungs, resulting in symptoms such as chronic bloody coughs, night sweats and weight loss, is the most common clinical manifestation of MTB infection. However, any organ in the body can be affected by spread of bacteria through the lymphatic system, causing disseminated or extra-pulmonary TB [2]. Extra-pulmonary TB can manifest itself as pericarditis, meningitis, or spinal TB [3].

Transmission of Mycobacterium tuberculosis occurs by inhalation of contaminated droplets released from the lungs of an infected individual, typically through coughing. Upon inhalation, the bacterium is ingested by means of phagocytosis by resident alveolar macrophages and tissue dendritic cells (DC), which are designed to kill pathogens but inside which MTB can subvert the killing mechanisms to allow replication [4].

MTB replicates very slowly, with a doubling time of about 24 hours. The bacterium measures around $0.5 \mu \mathrm{m}$ in diameter and $1-4 \mu \mathrm{m}$ in length, and is an aerobic intracellular pathogen [2]. There are other species which include bovis; the causative organism of TB in cows and rarely in humans. Others belonging to the Mycobacterium genus include Mycobacterium avium which causes a TB-like disease especially prevalent in patients in the advanced stages of Acquired Immune Deficiency Syndrome (AIDS), Mycobacterium leprae, the causative agent of leprosy as well as Mycobacterium smegmatis is non-pathogenic and very useful for the research analysis of other species in the genus Mycobacteria in cell culture laboratories among others [5].

Mycobacterium smegmatis, a soil dwelling saprophyte is distantly related to M. tuberculosis, is a mycobacterium model that is used to understand the pathogenesis of $M$. tuberculosis because of the disadvantages in the direct study of the mycobacterium. One disadvantage is that $M$. tuberculosis is a Category 3 human pathogen, requiring biosafety level III laboratory and animal facilities, substantial training before handling, and carries with it a risk of accidental exposure [6]. Secondly, $M$. tuberculosis grows slowly and colony formation requires two to three weeks, making its utilization for experimentation, time consuming [7]. Apart from its avirulent nature, allowing for the use of biosafety one laboratory, M. smegmatis is also fast growing and colony generation occurs in two to three days. According to Barry 2009 [8], 12 out of 19 M. tuberculosis virulence genes described to date share closely related homologues in $M$. smegmatis. Further, to determine the usefulness of M. smegmatis as an anti-tubercular drug discovery model, Altaf et al. 2010 [9] quantified the efficiency of M. smegmatis in detecting compounds that are inhibitory towards $M$. tuberculosis in compound library screening. From their results, M. smegmatis clearly illustrated usefulness in tuberculosis drug discovery.

The key to successful elimination of tuberculosis (TB) is treatment of cases with optimum chemotherapy. Isoniazid (INH), rifampin (RIF), ethambutol (EMB), pyrazinamide (PZA) and streptomycin (STR) are the essential first-line anti-tuberculosis drugs. Second-line anti-tuberculosis drugs include Aminoglycosides (kanamycin), quinolones (ciprofloxacilin), ethionamide, among others. The major problem of global concern is the emergence of resistance to existing medications by the causative organism. There have however been urgent calls for the inclusion of antimicrobials with some in vitro anti-tuberculosis (anti-TB) activity as part of the recommended drugs for the treatment to help solve the problems of high cost of therapy, inaccessibility of anti-TB drugs as well as bacterial resistance to existing medications. This motivated our interest to screen, in vitro, various anti-infective agents for effect against M. smegmatis.

\section{Materials and Methods}

\subsection{Test Organism}

The test organism, Mycobacterium smegmatis (MC2 155) was a gift from Noguchi Memorial Institute, Legon, Ghana. Middlebrook 7H9 powder, nutrient agar, and all reagents used for experiments were purchased from VWR, U.S.A. A loop full of a $24 \mathrm{hr}$ culture of $M$. smegmatis was transferred into sterile water and enumeration was done using the counting chamber method.

\subsection{Drugs}

Drug standards were obtained as gifts from the Centers for Disease Control, Atlanta, GA, USA. All other antimicrobials were purchased as tablets, capsules, or injectables from various pharmacies in Ghana and the United States of America. Drugs were prepared initially as $4 \mathrm{mg} / \mathrm{ml}$ stock in $10 \%$ Dimethyl sulfoxide (DMSO) solution. 
Final drug working solutions were prepared in Middlebrook 7H9 with 1\% DMSO.

\subsection{Agar Diffusion Method-Individual Drug Solutions}

$25 \mathrm{ml}$ nutrient agar portions were melted over boiling water at $100^{\circ} \mathrm{C}$ and stabilized in a water bath kept at $45^{\circ} \mathrm{C}$ prior to use. The stabilized agar was aseptically seeded with $100 \mu \mathrm{l}$ inoculum, equivalent to $2.0 \times 10^{6} \mathrm{cells} / \mathrm{ml}$ of Mycobacterium smegmatis and transferred into a sterile petri dish. Four wells were made in agar using a sterile \#7 cork borer.100 $\mu \mathrm{l}$ of various concentrations $(0.2-40 \mu \mathrm{g} / \mathrm{ml})$ of each anti-infective agent was transferred to each well, except for the last well which contained media without drug. The plates were prepared in triplicates with each plate serving as its own control. Negative control plates were prepared with only sterile media in the wells. The zones of inhibition (ZOI) were measured as the diameter of clear area without bacterial lawn around the edges of the wells. The minimum inhibitory concentration (MIC) was obtained as zero intercept of a linear regression of the diameter of these inhibition zones, $y$, plotted against the natural logarithm of the antibiotic concentration, $\mathrm{x}$ (Figure 1).

\subsection{Microdilution-Checkerboard Method}

Double strength (D/S) Middlebrook 7H9 media was used for this test. $1000 \mu \mathrm{l}$ of D/S Middlebrook broth was pipetted into each well of 24-wellmicrodilution plates. $200 \mu \mathrm{l}$ of the first anti-infective of the combination was put, in decreasing order of dilutions, into wells along the ordinate, while $200 \mu \mathrm{l}$ of the second drug was put into wells along the abscissa. $100 \mu \mathrm{l}$ each of the two drugs was put in the corresponding wells to produce drug combination volume of $200 \mu \mathrm{l}$ in varying concentrations. Each well was, then, filled with $700 \mu \mathrm{l}$ of media with $1 \%$ DMSO solution and inoculated with $100 \mu \mathrm{l}$ bacterial inoculum equivalent to $2.0 \times 10^{6} \mathrm{cells} / \mathrm{ml}$. Wells for positive control without any drug content and negative control wells without any inoculum were included on the plates. The resulting checkerboard contained each combination of two anti-infectives, with wells that contained the highest concentration of each antibiotic at opposite corners. The plates were incubated at $37^{\circ} \mathrm{C}$ for $24 \mathrm{hrs}$ under aerobic conditions. The MIC was determined as the lowest concentration of anti-infective that completely inhibited the growth of the organism as detected with the naked eye. The Fractional Inhibitory Concentration Index (FICI) was then calculated from the MIC of the individual drugs and combinations. Checkerboard assays result in a number of FIC indices. The sum of a number of FIC indices divided by the number of indices is designated as average, $\Sigma$ FIC. The $\Sigma$ FICs were calculated as follows: $\Sigma$ FIC $=$ FIC A + FIC B, where FIC A is the

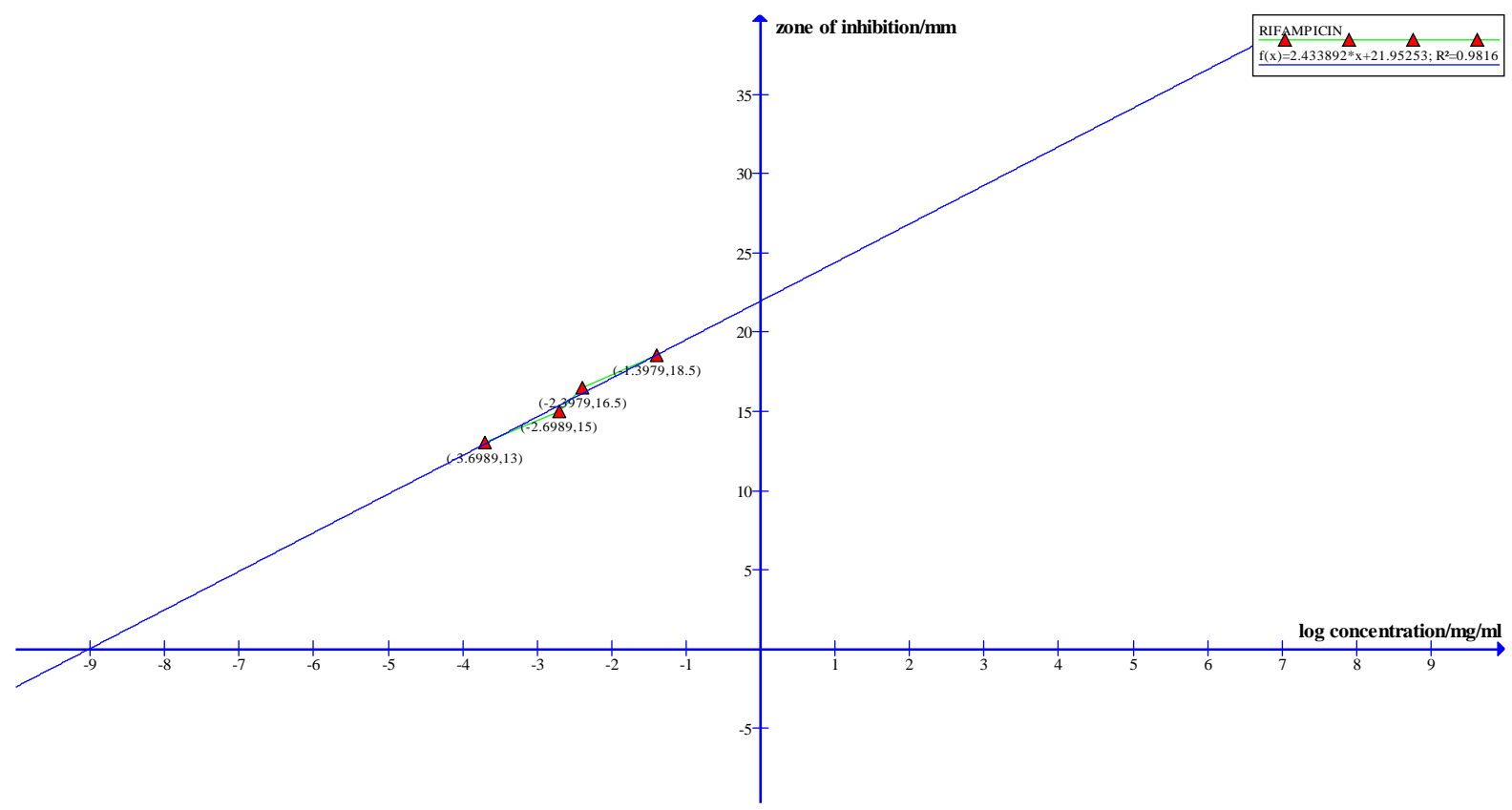


MIC of drug A in the combination/MIC of drug A alone, and FIC B is the MIC of drug B in the combination/MIC of drug B alone. The combination is considered synergistic when the $\Sigma F I C$ is $\leq 0.5$, additive when 0.5 $<\Sigma F I C<1$, indifferent when the $1<\Sigma F I C<2$, and antagonistic when the $\Sigma F I C$ is $\geq 2$ [10] [11].

\section{Results and Discussion}

\subsection{Results}

\subsubsection{Minimum Inhibitory Concentrations of Individual Anti-Infectives}

Agar diffusion method is a susceptibility test which gives an indication of the effectiveness of anti-infective agents against infections. In determining whether microbes are susceptible or not, the zones of inhibition are compared with that of reference ranges and an inference made. In the absence of reference ranges for the drug the MIC calculated for each drug is compared with the MIC of a standard anti-tuberculosis drug(s) used. For drug combinations, the MIC is compared with standard anti-TB drug combinations used in clinical practice. As such in the susceptibility determination a drug combination can be said to be effective against the mycobacterium if its MIC is equal to or lower than that of the standard combinations.

The MIC obtained for rifampicin, a first-line anti-TB drug was $4.16 \times 10^{-3} \mu \mathrm{g} / \mathrm{ml}$ per the agar diffusion method. Analysis of all other individual antimicrobials showed that meropenem, doripenem, ciprofloxacin, clarithromycin, erythromycin and clindamycin had considerably lower MIC than rifampicin (Table 1). Gentamicin, levofloxacin, tetracycline and piperacillin/tazobactam had MICs comparable to that of rifampicin. Imipenem, amikacin, cefaclor, doxycycline, dicloxacillin, azithromycin, and chloramphenicol had MICs much higher than that of rifampicin (Table 1). All the other first-line TB drugs, isoniazid, ethambutol and pyrazinamide were not active agents the strain of $M$. smegmatis used.

\subsubsection{Effect of Various Drug Combinations on M. smegmatis}

The standard anti-tuberculosis drug combination used was Rifampicin/Ethambutol. The MIC obtained for the combination was $8.37 \times 10^{-2} \mu \mathrm{g} / \mathrm{ml}$ as obtained from an agar diffusion method (data not included).The MIC's of each drug alone and in combination with a second drug were obtained (Table 2). In all the drug combinations, the MIC of drug A in combination with drug B was either approximately equal to or lower than the MIC of drug A alone. Likewise, the MIC of drug B in each combination was close to or lower than the MIC of drug B alone, except the clindamycin/amikacin combination where amikacin in the combination had a higher MIC than alone (Table 2).

\subsection{Discussion}

The mean zones of inhibition exhibited by the anti-infective agents used in the study indicate some level of susceptibility of Mycobacterium smegmatis to these agents even at concentrations lower than $2 \times 10^{-4} \mathrm{mg} / \mathrm{ml}$, which was the lowest concentration used. For a method such as broth dilution the lowest concentration at which the isolate is completely inhibited (as evidenced by the absence of visible bacterial growth) is recorded as MIC.

The lower MIC of the carbapenems, ciprofloxacin, clarithromycin, erythromycin and clindamycin as compared to that of rifampicin indicate that Mycobacterium smegmatis is susceptible to these agents and hence the agents can be described as effective against this specie. Ciprofloxacin is a 2nd line TB drug, whereas clarithromycin and erythromycin are 3rd line TB drugs; however the carbapenems are not indicated for the infection. The low MIC indicated for the carbapenems show that at much lower concentrations than rifampicin, they can be more effective in inhibiting the growth of Mycobacterium smegmatis and hence have promising efficacy against Mycobacterium species.

According to the broth dilution method, each individual drug was to some extent effective against $M$. smegmatis as observed from their MIC; however, the aim of drug combination of individual drugs is to achieve susceptibility with synergy. This is indicative of a good combination which can be developed for clinical use. The checkerboard method further tests the drug combinations and characterizes their susceptibility as synergistic, additive, indifferent or antagonistic. An indifferent effect of a combination of antibiotics is one that is equal to the effects of the most active component. The additive effect of a combination of antibiotics is one in which the effect of the combination is equal to that of the sum of the effects of the individual components. Synergistic effect of a combination of antibiotics is present if the effect of the combination exceeds the additive effects of 
Table 1. Minimum Inhibitory Concentrations (MIC) of various anti-infectives obtained by the Agar diffusion method as compared with the reference anti-TB drug-Rifampicin. NA = No Activity.

\begin{tabular}{|c|c|c|}
\hline Anti-Infective Agents & MIC (mg/ml) & MIC $(\mu \mathrm{g} / \mathrm{ml})$ \\
\hline Rifampicin (Reference) & $4.16 \mathrm{E}-06$ & $4.16 \mathrm{E}-03$ \\
\hline Meropenem & $1.58 \mathrm{E}-10$ & $1.58 \mathrm{E}-07$ \\
\hline Dorepenem & $2.23 \mathrm{E}-07$ & $2.23 \mathrm{E}-04$ \\
\hline Imipenem & $1.07 \mathrm{E}-04$ & $1.07 \mathrm{E}-01$ \\
\hline Piperacillin/Tazobactam & $3.47 \mathrm{E}-06$ & $3.47 \mathrm{E}-03$ \\
\hline Amikacin & $5.13 \mathrm{E}-05$ & $5.13 \mathrm{E}-02$ \\
\hline Gentamicin & $3.24 \mathrm{E}-06$ & $3.24 \mathrm{E}-03$ \\
\hline Cefaclor & $8.12 \mathrm{E}-03$ & 8.12 \\
\hline Ciprofloxacin & $6.17 \mathrm{E}-07$ & $6.17 \mathrm{E}-04$ \\
\hline Levofloxacin & $1.41 \mathrm{E}-06$ & $1.41 \mathrm{E}-03$ \\
\hline Doxycycline & $4.17 \mathrm{E}-05$ & $4.17 \mathrm{E}-02$ \\
\hline Dicloxacillin & $2.45 \mathrm{E}-05$ & $2.45 \mathrm{E}-02$ \\
\hline Tetracycline & $6.87 \mathrm{E}-06$ & $6.87 \mathrm{E}-03$ \\
\hline Azithromycin & $8.71 \mathrm{E}-05$ & $8.71 \mathrm{E}-02$ \\
\hline Clarithromycin & $7.24 \mathrm{E}-09$ & $7.24 \mathrm{E}-06$ \\
\hline Erythromycin & $1.99 \mathrm{E}-07$ & $1.99 \mathrm{E}-04$ \\
\hline Clindamycin & $9.55 \mathrm{E}-07$ & $9.55 \mathrm{E}-04$ \\
\hline Chloramphenicol & $1.25 \mathrm{E}-05$ & $1.25 \mathrm{E}-02$ \\
\hline Amoxicillin & NA & NA \\
\hline Amoxicillin/Clavulanate & NA & NA \\
\hline Ceftriaxone & NA & NA \\
\hline Flucloxacillin & NA & NA \\
\hline Penicillin G Sodium & NA & NA \\
\hline Nitrofurantoin & NA & NA \\
\hline Pentamidine & NA & NA \\
\hline Oseltamivir & NA & NA \\
\hline Quinine & NA & NA \\
\hline Isoniazid & NA & NA \\
\hline Ethambutol & NA & NA \\
\hline Pyrazinamide & NA & NA \\
\hline
\end{tabular}

Table 2. Minimum Inhibitory Concentrations (MIC) of Drug Combinations from broth dilution and the checkerboard method. $\mathrm{A}_{\mathrm{a}}$-MIC of drug $\mathrm{A}$ alone by broth dilution method, $\mathrm{A}_{\mathrm{c}}$-MIC of drug $\mathrm{A}$ in combination by checkerboard method, $\mathrm{B}_{\mathrm{a}}$-MIC of drug $\mathrm{B}$ alone by broth dilution method, $\mathrm{B}_{\mathrm{c}}$-MIC of drug $\mathrm{B}$ in combination by checkerboard method.

\begin{tabular}{ccccc}
\hline Drug combinations (A/B) & MIC (mg/ml) $\mathrm{A}_{\mathrm{a}}$ & MIC (mg/ml) $\mathrm{A}_{\mathrm{c}}$ & MIC (mg/ml) $\mathrm{B}_{\mathrm{a}}$ & MIC (mg/ml) $\mathrm{B}_{\mathrm{c}}$ \\
\hline Rif/Eth & $4 \times 10^{-2}$ & $1.0 \times 10^{-2}$ & $4 \times 10^{-1}$ & $5.0 \times 10^{-3}$ \\
Cip/Flu & $4 \times 10^{-3}$ & $2.5 \times 10^{-4}$ & $4 \times 10^{-1}$ & $1.0 \times 10^{-2}$ \\
Ery/Dox & $4 \times 10^{-2}$ & $2.0 \times 10^{-2}$ & $4 \times 10^{-3}$ & $2.5 \times 10^{-4}$ \\
Cla/Nit & $4 \times 10^{-2}$ & $5.0 \times 10^{-3}$ & $4 \times 10^{-1}$ & $1.0 \times 10^{-2}$ \\
Azi/Amo & $4 \times 10^{-3}$ & $2.5 \times 10^{-4}$ & $4 \times 10^{-1}$ & $1.0 \times 10^{-1}$ \\
Lev/Dic & $4 \times 10^{-4}$ & $4.0 \times 10^{-4}$ & $4 \times 10^{-1}$ & $2.0 \times 10^{-1}$ \\
Gen/Cef & $4 \times 10^{-2}$ & $4.0 \times 10^{-2}$ & $4 \times 10^{-3}$ & $2.5 \times 10^{-4}$ \\
Ami/Cli & $4 \times 10^{-2}$ & $8.0 \times 10^{-3}$ & $4 \times 10^{-3}$ & $2.0 \times 10^{-2}$ \\
\hline
\end{tabular}


Table 3. Minimum Inhibitory Concentrations (MIC) of Drug Combinations using the checkerboard method and their interpretation according to the reference ranges. Synergism when $\Sigma F I C$ is $\leq 0.5$, additive when $\Sigma F I C$ is $>0.5-1$, indifferent when the $\Sigma F I C$ is $>1$ to $<2$, and antagonistic when the $\Sigma F I C$ is $\geq 2$ [10] [11].

\begin{tabular}{ccc}
\hline Drug Combinations & $\Sigma$ FIC & Interpretation \\
\hline Rif/Eth & 0.26 & Synergy \\
Cip/Flu & 0.30 & Synergy \\
Ery/Dox & 0.56 & Additive \\
Cla/Nit & 0.38 & Synergy \\
Azi/Amo & 0.30 & Synergy \\
Lev/Dic & 1.50 & Indifference \\
Gen/Cef & 1.06 & Indifference \\
Ami/Cli & 5.20 & Antagonism \\
\hline
\end{tabular}

the individual components whereas antagonism is present if a reduced effect of a combination of antibiotics is observed in comparison with the effect of the most effective individual substance [10].

From the characterization by the checkerboard method, combinations of Rifampicin/Ethambutol, Ciprofloxacin/Flucloxacillin, Clarithromycin/Nitrofurantoin and Azithromycin/Amoxicillin showed synergism. Erythromycin/Doxycycline showed an additive effect, combinations of Levofloxacin/Dicloxacillin and Gentamicin/ Cefaclor showed indifference whereas the combination of Amikacin/Clindamycin showed antagonism (Table 3).

Antibiotics exert their effects as either bacteriostatic agents or bactericidal agents. By definition, a "bacteriostatic" agent is one that prevents the growth of bacteria. In other words, it keeps them in the stationary phase of growth. On the other hand, "bactericidal" agents kill actively growing bacteria [12]. In such light, the drug combinations were chosen such that drugs of similar effectiveness, to the extent possible, would be combined to potentiate their effects.

However from the results of the checkerboard method, it is observed that combinations of Clarithromycin/ Nitrofurantoin and Azithromycin/Amoxicillin produced synergistic effects though they possessed different antibacterial effectiveness. Both Nitrofurantoin and Amoxicillin are considered bactericidal agents whereas the macrolides, such as Azithromycin and Clarithromycin are considered classic bacteriostatic drug classes [13]. Specific macrolides-Erythromycin, Azithromycin and Clarithromycin have been shown to have bactericidal activity, in vitro, against Streptococcus pyogenes and Streptococcus pneumonia [14]-[16]. As a result Clarithromycin and Azithromycin could have in combination exhibited bactericidal activity against M. smegmatis and thus producing the synergistic effect.

Notably the combination of Amikacin/Clindamycin showed a significant antagonistic effect with FICI of 5.20. An evaluation of Amikacin indicated that it was highly bactericidal for Mycobacterium tuberculosis [17]. Clindamycin, on the other hand, may be bactericidal in vitro depending on microorganism and growth factors though it is a known bacteriostatic agent [18] [19].

In terms of their mechanism of antibacterial action, Amikacin irreversibly binds to $30 \mathrm{~S}$ and $50 \mathrm{~S}$ ribosomal subunits to interfere with the initiation of bacterial protein synthesis [20]. Clindamycin blocks peptide bond formation and also inhibits bacterial protein synthesis by binding to the $50 \mathrm{~S}$ ribosomal units [21]. Both drugs exert their antibiotic effect via a similar mechanism of action-inhibition of $50 \mathrm{~S}$ ribosomal enzymes. Such similarity could have accounted for the drugs producing an antagonistic effect when in combination.

\section{Acknowledgements}

The entire research was funded by the Office of Research, Innovation and Development (ORID) of the University of Ghana.

\section{References}

[1] North, R.J. and Jung, Y.-J. (2004) Immunity to Tuberculosis. Annual Review of Immunology, 22, 599-623. http://dx.doi.org/10.1146/annurev.immunol.22.012703.104635 http://www.ncbi.nlm.nih.gov/pubmed/15032590

[2] Ducati, R.G., et al. (2006) The Resumption of Consumption-A Review on Tuberculosis. Memórias do Instituto Os- 
waldo Cruz, 101, 697-714. http://dx.doi.org/10.1590/S0074-02762006000700001

http://www.ncbi.nlm.nih.gov/pubmed/17160276

[3] Russell, D.G., et al. (2009) Foamy Macrophages and the Progression of the Human Tuberculosis Granuloma. Nature Immunology, 10, 943-948.

http://www.pubmedcentral.nih.gov/articlerender.fcgi?artid=2759071\&tool=pmcentrez\&rendertype=abstract

[4] Zignol, M., et al. (2006) Global Incidence of Multidrug-Resistant Tuberculosis. The Journal of Infectious Diseases, 194, 479-485. http://dx.doi.org/10.1086/505877 http://www.ncbi.nlm.nih.gov/pubmed/16845631

[5] Sareen, D., et al. (2003) Mycothiol Is Essential for Growth of Mycobacterium tuberculosis Erdman. Journal of Bacteriology, 185, 6736-6740. http://dx.doi.org/10.1128/JB.185.22.6736-6740.2003

http://www.pubmedcentral.nih.gov/articlerender.fcgi?artid=262099\&tool=pmcentrez\&rendertype=abstract

[6] Parish, T. and Stoker, N.G. (2001) Mycobacterium tuberculosis Protocols. Humana Press, Clifton. http://link.springer.com/10.1385/1592591477

[7] Shiloh, M.U. and Champion, P.A.D. (2010) To Catch a Killer. What Can Mycobacterial Models Teach Us about Mycobacterium tuberculosis Pathogenesis? Current Opinion in Microbiology, 13, 86-92. http://dx.doi.org/10.1016/j.mib.2009.11.006 http://www.sciencedirect.com/science/article/pii/S1369527409001775

[8] Barry, C.E., et al. (2009) The Spectrum of Latent Tuberculosis: Rethinking the Biology and Intervention Strategies. Nature Reviews Microbiology, 7, 845-855. http://dx.doi.org/10.1038/nrmicro2236

[9] Altaf, M., et al. (2010) Evaluation of the Mycobacterium smegmatis and BCG Models for the Discovery of Mycobacterium tuberculosis Inhibitors. Tuberculosis (Edinburgh, Scotland), 90, 333-337. http://dx.doi.org/10.1016/j.tube.2010.09.002 http://www.sciencedirect.com/science/article/pii/S1472979210001034

[10] EUCAST of ESCMID (2000) EUCAST Definitive Document Terminology Relating to Methods for the Determination of Susceptibility of Bacteria to Antimicrobial Agents. Clinical Microbiology and Infection, 6, 503- 508.

[11] Orhan, G., et al. (2005) Synergy Tests by E Test and Checkerboard Methods of Antimicrobial Combinations against Brucella melitensis. Journal of Clinical Microbiology, 43, 140-143. http://dx.doi.org/10.1128/JCM.43.1.140-143.2005 http://jcm.asm.org/content/43/1/140.full\#ref-3

[12] Pankey, G.A. and Sabath, L.D. (2004) Clinical Relevance of Bacteriostatic versus Bactericidal Mechanisms of Action in the Treatment of Gram-Positive Bacterial Infections. Clinical Infectious Diseases, 38, 864-870. http://dx.doi.org/10.1086/381972 http://cid.oxfordjournals.org/content/38/6/864.full

[13] Neal, M.J. (2010) Medical Pharmacology at a Glance, Custom. John Wiley \& Sons, Hoboken. http://books.google.com.gh/books/about/Medical_Pharmacology_at_a_Glance_Custom.html?id=mjBaK0o0SIwC\&pgi $\underline{\mathrm{s}=1}$

[14] Fernandes, P.B., et al. (1986) In Vitro and in Vivo Evaluation of A-56268 (TE-031), a New Macrolide. Antimicrobial agents and Chemotherapy, 30, 865-873. http://dx.doi.org/10.1128/AAC.30.6.865 http://www.pubmedcentral.nih.gov/articlerender.fcgi?artid=180609\&tool=pmcentrez\&rendertype=abstract

[15] Haight, T.H. and Finland, M. (1952) Observations on Mode of Action of Erythromycin. Proceedings of the Society for Experimental Biology and Medicine. Society for Experimental Biology and Medicine, 81, 188-193. http://dx.doi.org/10.3181/00379727-81-19817 http://www.ncbi.nlm.nih.gov/pubmed/13047353

[16] Piscitelli, S.C., Danziger, L.H. and Rodvold, K.A. (1992) Clarithromycin and Azithromycin: New Macrolide Antibiotics. Clinical Pharmacy, 11, 137-152. http://www.ncbi.nlm.nih.gov/pubmed/1312921

[17] Heifets, L. and Lindholm-Levy, P. (1989) Comparison of Bactericidal Activities of Streptomycin, Amikacin, Kanamycin, and Capreomycin against Mycobacterium avium and M. Tuberculosis. Antimicrobial Agents and Chemotherapy, 33, 1298-1301. http://dx.doi.org/10.1128/AAC.33.8.1298

[18] Nastro, L.J. and Finegold, S.M. (1972) Bactericidal Activity of Five Antimicrobial Agents against Bacteroides fragilis. Journal of Infectious Diseases, 126, 104-107. http://dx.doi.org/10.1093/infdis/126.1.104 http://jid.oxfordjournals.org/content/126/1/104.abstract?ijkey=0bf210afc1a0bce6e48e57950037e366e1510232\&keytyp $\underline{\mathrm{e} 2=\mathrm{tf} \text { ipsecsha }}$

[19] Sande, M.A. and Johnson, M.L. (1975) Antimicrobial Therapy of Experimental Endocarditis Caused by Staphylococcus aureus. Journal of Infectious Diseases, 131, 367-375. http://dx.doi.org/10.1093/infdis/131.4.367 http://jid.oxfordjournals.org/content/131/4/367.abstract?ijkey=85e9131cb05e5ed9f5aa9b922cd7c67eeb8ad535\&keytyp e2=tf_ipsecsha

[20] Eghianruwa, K. (2014) Essential Drug Data for Rational Therapy in Veterinary Practice (Google eBook). AuthorHouse UK. http://books.google.com/books?id=CtfIAgAAQBAJ\&pgis=1

[21] Staines, H.M. and Krishna, S. (2012) Treatment and Prevention of Malaria: Antimalarial Drug Chemistry, Action and Use. Springer Basel AG, Basel. http://books.google.com/books?id=cNuY6tyyyrUC\&pgis=1 
Scientific Research Publishing (SCIRP) is one of the largest Open Access journal publishers. It is currently publishing more than 200 open access, online, peer-reviewed journals covering a wide range of academic disciplines. SCIRP serves the worldwide academic communities and contributes to the progress and application of science with its publication.

Other selected journals from SCIRP are listed as below. Submit your manuscript to us via either submit@scirp.org or Online Submission Portal.
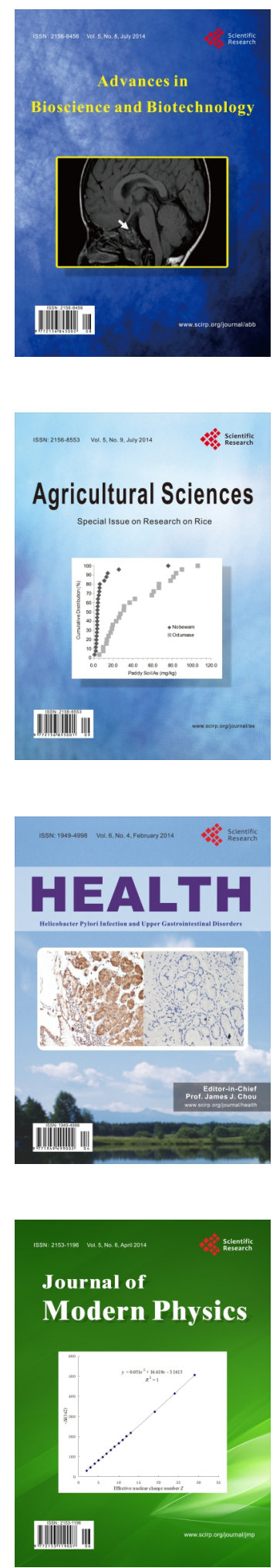
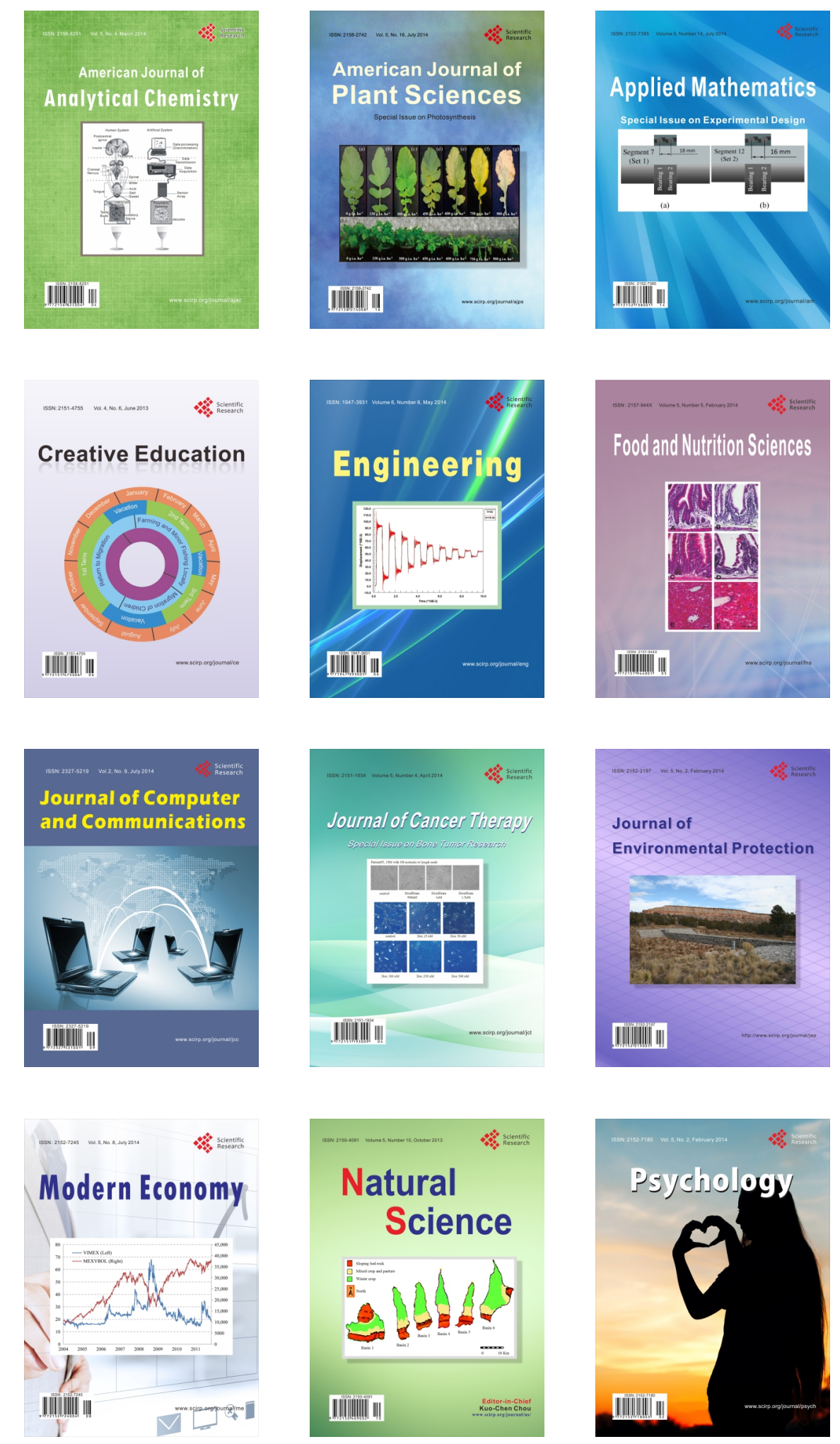